\title{
Invariant Polynomial Functions on $k$ qudits
}

\author{
Jean-Luc Brylinski*† Ranee Brylinski ${ }^{\ddagger}$
}

November 5, 2018

\begin{abstract}
We study the polynomial functions on tensor states in $\left(\mathbb{C}^{n}\right)^{\otimes k}$ which are invariant under $S U(n)^{k}$. We describe the space of invariant polynomials in terms of symmetric group representations. For $k$ even, the smallest degree for invariant polynomials is $n$ and in degree $n$ we find a natural generalization of the determinant. For $n, d$ fixed, we describe the asymptotic behavior of the dimension of the space of invariants as $k \rightarrow \infty$. We study in detail the space of homogeneous degree 4 invariant polynomial functions on $\left(\mathbb{C}^{2}\right)^{\otimes k}$.
\end{abstract}

\section{Introduction}

In quantum mechanics, a combination of states in Hilbert spaces $H_{1}, . ., H_{k}$ leads to a state in the tensor product Hilbert space $H_{1} \otimes \cdots \otimes H_{k}$. Such a state will be called here a tensor state. In this paper we take $H_{1}=\cdots=H_{k}=\mathbb{C}^{n}$ where $n>1$. Then a tensor state is a joint state of $k$ qudits. It would be very interesting to classify tensor states in $\left(\mathbb{C}^{n}\right)^{\otimes k}$ up to the action of the product $U(n)^{k}$ of unitary groups of local symmetries. A natural approach to this is to study the algebra of invariant polynomials. This approach was developed by Rains [R], by Grassl, Rötteler and Beth [G-R-B1] G-R-B2], by Linden and Popescu [L-P] and by Coffman, Kundu and Wootters [C-K-W]. These authors study the ring of invariant polynomials in the components of a tensor state in $\left(\mathbb{C}^{n}\right)^{\otimes k}$ and in their complex-conjugates. For $k$ qubits, explicit descriptions of invariants are given in G-R-B1, G-R-B2, [L-P and in [C-K-W].

In this paper the symmetry group we consider is the product $G=S U(n)^{k}$ of special unitary groups; one thinks of $G$ as the special group of local symmetries. We study the $G$-invariant polynomial functions $Q$ on the tensor states in $\left(\mathbb{C}^{n}\right)^{\otimes k}$ (we discuss in \$2 how this is relevant to the description of the $G$-orbits). We consider polynomials in the entries of a tensor state, in other words, holomorphic polynomials.

\footnotetext{
*Department of Mathematics, Penn State University, University Park 16802, jlb@math.psu.edu

${ }^{\dagger}$ Research supported in part by NSF Grant No. DMS-9803593

${ }_{\ddagger}^{\ddagger}$ Department of Mathematics, Penn State University, University Park 16802, rkb@math.psu.edu
} 
Let $\mathcal{R}_{n, k, d}$ be the space of homogeneous degree $d$ polynomial functions on tensor states in $\left(\mathbb{C}^{n}\right)^{\otimes k}$. Let $\mathcal{R}_{n, k, d}^{G}$ be the space of $G$-invariants in $\mathcal{R}_{n, k, d}$. See $\S \Omega$ for more discussion. We reduce the problem of computing $\mathcal{R}_{n, k, d}^{G}$ to a problem in the invariant theory of the symmetric group $\mathfrak{S}_{d}$ (Proposition 2.1). In particular, $\mathcal{R}_{n, k, d}^{G}$ is non-zero only if $d$ is a multiple of $n$. So the "first" case is $d=n$; we examine this in $\$$. 3 . We find that if $k$ is odd then $\mathcal{R}_{n, k, n}^{G}=0$ while if $k$ is even then $\mathcal{R}_{n, k, n}^{G}$ is 1-dimensional. In the latter case we write down (\$3) explicitly the corresponding invariant polynomial $P_{n, k}$ in $\mathcal{R}_{n, k, n}$; we find $P_{n, k}$ is a natural generalization of the determinant of a square matrix.

For fixed $n, d$ the direct sum $\oplus_{k} \mathcal{R}_{n, k, d}$ is an associative algebra. We study the asymptotic behavior of $\operatorname{dim} \mathcal{R}_{n, k, d}^{G}$ as $k \rightarrow \infty$ in 䧃. In 的, we specialize to the case of $k$-qubits, i.e. $n=2$. We compute the dimension of the space $\mathcal{R}_{2, k, 4}^{G}$ of degree 4 invariants as well as the dimension of the space of invariants in $\mathcal{R}_{2, k, 4}^{G}$ under the natural action of $\mathfrak{S}_{k}$. We show that $\oplus_{k} \mathcal{R}_{2, k, 4}^{\mathfrak{S}_{k} \mathfrak{K} G}$ is a polynomial algebra on 2 generators. For $k \leq 5$ we describe the representation of $\mathfrak{S}_{k}$ on $\mathcal{R}_{2, k, 4}^{G}$. For $k=4$ we find some interesting relations with the results on classification of tensor states in $\left(\mathbb{C}^{2}\right)^{\otimes 4}$ given in $\left.\mathbb{B}\right]$.

We thank Markus Grassl for his useful comments on the first version of this paper.

\section{Polynomial invariants of tensor states}

We will consider $\left(\mathbb{C}^{n}\right)^{\otimes k}$ as a space of contravariant tensor states $u$. Then (once we fix a basis of $\left.\mathbb{C}^{n}\right) u$ is given by $n^{k}$ components $u^{p_{1} p_{2} \cdots p_{k}}$. We consider the algebra $\mathcal{R}_{n, k}$ of polynomial functions on $\left(\mathbb{C}^{n}\right)^{\otimes k}$. So $\mathcal{R}_{n, k}$ is the polynomial algebra $\mathbb{C}\left[x_{p_{1} p_{2} \cdots p_{k}}\right]$ in the $n^{k}$ coordinate functions $x_{p_{1} p_{2} \cdots p_{k}}$. We have a natural algebra grading $\mathcal{R}_{n, k}=\oplus_{d=0}^{\infty} \mathcal{R}_{n, k, d}$ where $\mathcal{R}_{n, k, d}$ is the space of homogeneous degree $d$ polynomial functions.

A function in $\mathcal{R}_{n, k, d}$ amounts to a symmetric degree $d$ covariant tensor $Q$ in $\left(\mathbb{C}^{n}\right)^{\otimes k}$. So $Q$ has $n^{d k}$ components $Q_{i_{11} \cdots i_{d k}}$ where we think of the indices $i_{a b}$ as being arranged in a rectangular array of $d$ rows and $k$ columns and $Q_{i_{11} \cdots i_{d k}}$ is invariant under permutations of the rows of the array. Then $Q$ defines the function

$$
u \mapsto Q_{i_{11} \cdots i_{d k}} u^{i_{11} i_{12} \cdots i_{1 k}} u^{i_{21} i_{22} \cdots i_{2 k}} \cdots u^{i_{d 1} i_{d 2} \cdots i_{d k}}
$$

where we used the usual Einstein summation convention. In this way, $\mathcal{R}_{n, k}$ identifies with $S^{d}\left(\left(\mathbb{C}^{n}\right)^{\otimes k}\right)$.

Now the group $G=S U(n)^{k}$ acts on our tensor states $u$ and tensors $Q$ as follows. Let the matrix $g_{i j}$ live in the $m$-th copy of $S U(n)$ and let $g^{i j}$ be the inverse matrix. Then $g_{i j}$ transforms $u^{p_{1} p_{2} \cdots p_{k}}$ into $g_{p_{m} q_{m}} u^{q_{1} q_{2} \cdots \cdots q_{k}}$ and $Q_{i_{11} \cdots i_{d k}}$ into $Q_{j_{11} \cdots j_{d k}} g^{j_{1 m} i_{1 m}} g^{j_{2 m} i_{2 m}} \cdots g^{j_{d m} i_{d m}}$. The identification of $\mathcal{R}_{n, k, d}$ with $S^{d}\left(\left(\mathbb{C}^{n}\right)^{\otimes k}\right)$ is $G$-equivariant.

We are interested in the algebra $\mathcal{R}_{n, k}^{G}=\oplus_{d=0}^{\infty} \mathcal{R}_{n, k, d}^{G}$ of $G$-invariants. We view this as a first step towards studying the orbits of $G$ on $\left(\mathbb{C}^{n}\right)^{\otimes k}$. One can first study the orbits of the complex group $G_{\mathbb{C}}=S L(n, \mathbb{C})^{k}$ and then decompose the $G_{\mathbb{C}^{-}}$orbits under the $G$-action. Note that a polynomial is $G$-invariant if and only if its is $G_{\mathbb{C}}$-invariant. The closed $G_{\mathbb{C}}$ orbits play a special role - they are the most degenerate orbits. Given any orbit $Y$, its closure contains a unique closed orbit $Z$; then points in $Y$ degenerate to 


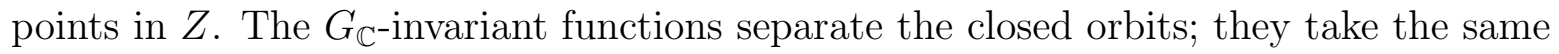
values on $Y$ and on $Z$. The set of closed orbits of $G_{\mathbb{C}}$ in $\left(\mathbb{C}^{n}\right)^{\otimes k}$ has the structure of an affine complex algebraic variety with $\mathcal{R}_{n, k}^{G}$ as its algebra of regular functions. Thus a complete description of $\mathcal{R}_{n, k}^{G}$ would lead to a precise knowledge of the closed $G_{\mathbb{C}^{-}}$-orbits.

Our approach is thus somewhat different from that of [R] [G-R-B1] [G-R-B2] [L-P] C-K-W who study the invariant functions on $\left(\mathbb{C}^{n}\right)^{\otimes k}$ which are polynomials in the $x_{p_{1} \cdots p_{k}}$ and in their complex conjugates; these can also be described as the invariant polynomial functions on $\left(\mathbb{C}^{n}\right)^{\otimes k} \oplus \overline{\left(\mathbb{C}^{n}\right)^{\otimes k}}$.

At this point it is useful to examine the case $k=2$. We can identify $\left(\mathbb{C}^{n}\right)^{\otimes 2}$ with the space $M_{n}(\mathbb{C})$ of square matrices and then $G=S U(n)^{2}$ acts on $M_{n}(\mathbb{C})$ by $(g, h) \cdot u=g u h^{-1}$. So $\mathcal{R}_{n, k, d}^{G}$ is the space of homogeneous degree $d$ polynomial functions $Q$ of an $n$ by $n$ matrix $u$ which are bi-SL(n, $\mathbb{C})$-invariant, i.e. $Q\left(g u h^{-1}\right)=Q(u)$ for $g, h \in S L(n, \mathbb{C})$. Then $Q$ is, up to scaling, the $r$ th power of the determinant $D$ for some $r$. Hence $d=r n$. It follows that $\mathcal{R}_{n, 2}^{G}$ is the polynomial algebra $\mathbb{C}[D]$. Thus the space of closed orbits for $S L(n, \mathbb{C})^{2}$ identifies with $\mathbb{C}$, where $\lambda$ corresponds to the unique closed orbit $Z_{\lambda}$ inside the set $X_{\lambda}$ of matrices of determinant $\lambda$. For $\lambda \neq 0, Z_{\lambda}=X_{\lambda}$ while for $\lambda=0, Z_{0}$ reduces to the zero matrix.

We view $S^{d}\left(\left(\mathbb{C}^{n}\right)^{\otimes k}\right)$ as the space of invariants for the symmetric group $\mathfrak{S}_{d}$ acting on $\left(\left(\mathbb{C}^{n}\right)^{\otimes k}\right)^{\otimes d}$. So

$$
\mathcal{R}_{n, k, d}^{G}=\left(\left(\left(\mathbb{C}^{n}\right)^{\otimes k}\right)^{\otimes d}\right)^{G \times \mathfrak{S}_{d}}=\left(\left(\left(\mathbb{C}^{n}\right)^{\otimes d}\right)^{\otimes k}\right)^{G \times \mathfrak{S}_{d}}
$$

Recall the Schur decomposition $\left(\mathbb{C}^{n}\right)^{\otimes d}=\oplus_{\alpha} S^{\alpha}\left(\mathbb{C}^{n}\right) \otimes E_{\alpha}$ where $\alpha$ ranges over partitions of $d$ with at most $n$ rows, $S^{\alpha}\left(\mathbb{C}^{n}\right)$ is the irreducible covariant representation of $S U(n)$ given by the Schur functor $S^{\alpha}$, and $E_{\alpha}$ is the corresponding irreducible representation of $\mathfrak{S}_{d}$. We use the convention that $E_{\alpha}$ is the trivial representation if $\alpha=[d]$, while $E_{\alpha}$ is the sign representation if $\alpha=\left[1^{d}\right]$. Thus we have

$$
\left(\left(\mathbb{C}^{n}\right)^{\otimes d}\right)^{\otimes k}=\sum_{\left|\alpha_{1}\right|=\cdots=\left|\alpha_{k}\right|=d} S^{\alpha_{1}}\left(\mathbb{C}^{n}\right) \otimes \cdots \otimes S^{\alpha_{k}}\left(\mathbb{C}^{n}\right) \otimes E_{\alpha_{1}} \otimes \cdots \otimes E_{\alpha_{k}}
$$

Now taking the invariants under $G \times \mathfrak{S}_{d}$ we get

$$
\mathcal{R}_{n, k, d}^{G}=\sum_{\left|\alpha_{1}\right|=\cdots=\left|\alpha_{k}\right|=d} S^{\alpha_{1}}\left(\mathbb{C}^{n}\right)^{S U(n)} \otimes \cdots \otimes S^{\alpha_{k}}\left(\mathbb{C}^{n}\right)^{S U(n)} \otimes\left(E_{\alpha_{1}} \otimes \cdots \otimes E_{\alpha_{k}}\right)^{\mathfrak{S}_{d}}
$$

The representation $S^{\alpha_{j}}\left(\mathbb{C}^{n}\right)$, since it is irreducible, has no $S U(n)$-invariants except if $S^{\alpha_{j}}\left(\mathbb{C}^{n}\right)=\mathbb{C}$ is trivial. This happens if and only if $\alpha_{j}$ is a rectangular partition with all columns of length $n$. This proves:

Proposition 2.1. If $n$ does not divide $d$, then $\mathcal{R}_{n, k, d}^{G}=0$. If $d=n r$, then $\mathcal{R}_{n, k, d}^{G}$ is isomorphic to $\left(E_{\pi}^{\otimes k}\right)^{\mathfrak{S}_{d}}$ where $\pi=\left[r^{n}\right]$.

The permutation action of $\mathfrak{S}_{k}$ on $\left(\mathbb{C}^{n}\right)^{\otimes k}$ induces an action of $\mathfrak{S}_{k}$ on $\mathcal{R}_{n, k, d}^{G}$.

Corollary 2.2. The isomorphism of Proposition 2.1 intertwines the $\mathfrak{S}_{k}$-action on $\mathcal{R}_{n, k, d}^{G}$ with the action of $\mathfrak{S}_{k}$ on $\left(E_{\pi}^{\otimes k}\right)^{\mathfrak{S}_{d}}$ given by permuting the $k$ factors $E_{\pi}$. 


\section{The generalized determinant function}

Given $n$ and $k$, we want to find the smallest positive value of $d$ such that $\mathcal{R}_{n, k, d}^{G} \neq 0$. By Proposition 2.1, the first candidate is $d=n$.

Corollary 3.1. $\mathcal{R}_{n, k, n}^{G} \neq 0$ iff $k$ is even. In that case, $\mathcal{R}_{n, k, n}^{G}$ is one-dimensional and consists of the multiples of the function $P_{n, k}$ given by

$$
P_{n, k}(u)=\sum_{\sigma_{2}, \cdots, \sigma_{k} \in \mathfrak{S}_{n}} \epsilon\left(\sigma_{2}\right) \cdots \epsilon\left(\sigma_{k}\right) \prod_{h=1}^{n} u^{h h_{\sigma_{2}} \cdots h_{\sigma_{k}}}
$$

where $h_{\sigma_{j}}=\sigma_{j}(h)$.

Proof. By Proposition 2.1, we need to compute $\left(E_{\pi}^{\otimes k}\right)^{\mathfrak{S}_{d}}$. For $d=n, \pi=\left[1^{n}\right]$ and so $E_{\pi}$ is the sign representation of $\mathfrak{S}_{n}$. Then $\left(E_{\pi}^{\otimes k}\right)$ is one-dimensional and carries the trivial representation if $k$ is even, or the sign representation if $k$ is odd.

Now for $k$ even, we can easily compute a non-zero function $P=P_{n, k}$ in $\mathcal{R}_{n, k, n}$. For $S^{\pi}\left(\mathbb{C}^{n}\right)$ is the top exterior power $\wedge^{n} \mathbb{C}^{n}$. Thus $P$ is a non-zero element of the onedimensional subspace $\left(\wedge^{n} \mathbb{C}^{n}\right)^{\otimes k}$ of $\left(\left(\mathbb{C}^{n}\right)^{\otimes n}\right)^{\otimes k}$. The tensor components of $P$ are then given by $P_{i_{11} \cdots i_{n k}}=\frac{1}{n !} \epsilon\left(\sigma_{1}\right) \cdots \epsilon\left(\sigma_{k}\right)$ if for each $j$, the column $i_{1 j}, \cdots, i_{n j}$ is a permutation $\sigma_{j}$ of $1, \cdots, n$ and 0 otherwise. Then we get

$$
P_{n, k}(u)=\frac{1}{n !} \sum_{\sigma_{1}, \cdots, \sigma_{k} \in \mathfrak{S}_{n}} \epsilon\left(\sigma_{1}\right) \cdots \epsilon\left(\sigma_{k}\right) \prod_{h=1}^{n} u^{h_{\sigma_{1}} \cdots h_{\sigma_{k}}}
$$

where $h_{\sigma_{i}}=\sigma_{i}(h)$. The expression is very redundant, as each term appears $n$ ! times. We remedy this by restricting the first permutation $\sigma_{1}$ to be 1 . This gives (3.1).

$P_{n, k}$ is a generalized determinant; $P_{n, k}$ is invariant under the $\mathfrak{S}_{k}$-action. For $k=2$, (3.1) reduces to the usual formula for the matrix determinant.

Recall that the rank $s$ of a tensor state $u$ in $\left(\mathbb{C}^{n}\right)^{\otimes k}$ is the smallest integer $s$ such that $u$ can be written as $u=v_{1}+v_{2}+\cdots+v_{s}$, where the $v_{i}$ are decomposable tensor states $v_{i}=w_{i 1} \otimes w_{i 2} \otimes \cdots \otimes w_{i k}$. There is a relation between the rank and the vanishing of $P_{n, k}$ as follows:

Corollary 3.2. If the tensor state $u$ in $\left(\mathbb{C}^{n}\right)^{\otimes k}$ has rank less than $n$, then $P_{n, k}(u)=0$.

It is easy to find a tensor state $u$ of rank $n$ such that $P_{n, k}(u)$ is non-zero. For instance, $P_{n, k}(u)=1$ if $u$ has all components zero except $u^{1 \cdots 1}=\cdots=u^{n \cdots n}=1$. For $k=2, P_{n, k}(u)=0$ implies $u$ has rank less than $n$. For bigger (even) $k$, this is false, if $n$ is large enough. This happens essentially because the rank of $u$ can be very large (at least $\frac{n^{k}}{k n-k+1}$ ). Thus $P_{n, k}$ gives only partial information about the rank.

\section{Asymptotics as $k \rightarrow \infty$}

Suppose we fix $n$ and $d$ where $d=r n$. Then there is a $G$-invariant associative graded algebra structure $P \circ Q$ on the direct sum $\oplus_{k} \mathcal{R}_{n, k, d}^{G}$. Indeed, the product of tensors 
induces a $\left(G \times \mathfrak{S}_{d}\right)$-invariant map $V^{\otimes k} \otimes V^{\otimes l} \rightarrow V^{\otimes(k+l)}$ where $V=\left(\mathbb{C}^{n}\right)^{\otimes d}$. The induced multiplication on the spaces of $\left(G \times \mathfrak{S}_{d}\right)$-invariants gives the product on $\oplus_{k} \mathcal{R}_{n, k, d}^{G}$, where we use the identification in (2.2). This multiplication corresponds, under the isomorphism of Proposition 2.1, to the product map $E_{\pi}^{\otimes k} \otimes E_{\pi}^{\otimes l} \rightarrow E_{\pi}^{\otimes(k+l)}$. This structure is very useful. For instance, if $d=n$, then $P_{n, k} \circ P_{n, l}=\frac{1}{n !} P_{n, k+l}$. Thus the determinant $P_{n, 2}$ determines $P_{n, 2 m}$ in that the $m$-fold product $P_{n, 2} \circ \cdots \circ P_{n, 2}$ is equal to $(n !)^{-m+1} P_{n, 2 m}$.

We will study the size of the algebra $\oplus_{k} \mathcal{R}_{n, k, d}^{G}$ by finding an asymptotic formula for the dimension of $\mathcal{R}_{n, k, d}^{G}$. We do this for $r \geq 2$. Indeed for $r=1$ we already know $\operatorname{dim} \mathcal{R}_{n, k, n}^{G}$ is 1 if $k$ is even or 0 if $k$ is odd; we call this the static case. The asymptotics involve the number

$$
p=\operatorname{dim} E_{\pi}=d ! \prod_{m=0}^{n-1} \frac{m !}{(m+r) !}
$$

where $\pi=\left[r^{n}\right]$ as in Proposition 2.1. Our formula for $p$ is immediate from the hook formula for the dimension of an irreducible symmetric group representation.

Proposition 4.1. Assume $d=r n$ with $r \geq 2$. Then $\operatorname{dim} \mathcal{R}_{n, k, d}^{G} \sim c \frac{p^{k}}{d !}$ as $k \rightarrow \infty$, where $c=1$ with one exception: $c=4$ if $n=2, d=4$.

Proof. Let $s=\operatorname{dim} \mathcal{R}_{n, k, d}^{G}=\operatorname{dim}\left(E_{\pi}^{\otimes k}\right)^{\mathfrak{S}_{d}}$. Then $s=\frac{1}{d !} \sum_{\sigma \in \mathfrak{S}_{d}} \chi(\sigma)^{k}$ where $\chi: \mathfrak{S}_{d} \rightarrow \mathbb{Z}$ is the character of $E_{\pi}$. If $\sigma$ acts trivially on $E_{\pi}$, then $\chi(\sigma)=p$. If $\sigma$ acts non-trivially, we claim $|\chi(\sigma)|<p$. To show this, it suffices to show that $\sigma$ has at least two distinct eigenvalues on $E_{\pi}$; this is because $\chi(\sigma)$ is the sum of the $p$ eigenvalues of $\sigma$. Now the set $\mathfrak{T}_{d}$ of $\sigma \in \mathfrak{S}_{d}$ which act on $E_{\pi}$ by a scalar is a normal subgroup of $\mathfrak{S}_{d}$. So if $d \geq 5$, then $\mathfrak{T}_{d}$ is $\{1\}$, the alternating group $\mathfrak{A}_{d}$ or $\mathfrak{S}_{d}$. We can easily rule out the latter two possibilities, so $\mathfrak{T}_{d}=\{1\}$, which proves our claim. If $d \leq 4$, then (since $r>1$ and $n>1$ ), we have $d=4, n=2$ and $\pi=[2,2]$. Our claim is clear here since $\mathfrak{S}_{4}$ acts on $E_{\pi}$ through the reflection representation of $\mathfrak{S}_{3}$ on $\mathbb{C}^{2}$.

Therefore we have $s=c \frac{p^{k}}{d !}+o\left(p^{k}\right)$ as $k \rightarrow \infty$ where $c$ is cardinality of the kernel of $\mathfrak{S}_{d} \rightarrow$ Aut $E_{\pi}$. Our work in the previous paragraph computes $c$.

Proposition 4.1 implies that the algebra $\oplus_{k} \mathcal{R}_{n, k, d}^{G}$ is far from commutative, as it has roughly $1 / N$ times the size of the tensor algebra $\oplus_{k}\left(\mathbb{C}^{p}\right)^{\otimes k}$. We note however that the $\mathfrak{S}_{k}$-invariants in $\oplus_{k} \mathcal{R}_{n, k, d}^{G}$ form a commutative subalgebra, isomorphic to $S\left(E_{\pi}\right)^{\mathfrak{S}_{d}}$.

\section{$5 \quad$ Quartic invariants of $k$ qubits}

The case $n=2$ is of particular interest, as here the qudits are qubits, and this is the case being most discussed in quantum computation. Here we can give some precise nonasymptotic results for the first non-static case, namely $\mathcal{R}_{2, k, 4}^{G}$. We put $E=E_{\pi}=E_{[2,2]}$. The proof of Proposition 4.1 easily gives

Corollary 5.1. We have $\operatorname{dim} \mathcal{R}_{2, k, 4}^{G}=\frac{1}{3}\left(2^{k-1}+(-1)^{k}\right)$. 
The first few values of $\operatorname{dim} \mathcal{R}_{2, k, 4}^{G}$, starting at $k=1$, are $0,1,1,3,5,11,21,43$. For $k=2$ and $k=3$ the unique (up to scalar) invariants are, respectively, the squared determinant $P_{2,2}^{2}$ and the Cayley hyperdeterminant $H_{2,3}$ (see [G-K-Z]). We note that the hyperdeterminant is very closely related to the relative tangle of 3 entangled qubits discussd in [C-K-W].

It would be useful to study $\mathcal{R}_{2, k, 4}^{G}$ as a representation of $\mathfrak{S}_{k}$, where $\mathfrak{S}_{k}$ acts by permuting the $k$ qubits. The $\mathfrak{S}_{k}$-invariants in $\mathcal{R}_{2, k, 4}^{G}$ are the $\left(\mathfrak{S}_{k} \times G\right)$-invariants in $\mathcal{R}_{2, k, 4}$. These $\left(\mathfrak{S}_{k} \ltimes G\right)$-invariant polynomials are very significant as they separate the closed orbits of the extended symmetry group $\mathfrak{S}_{k} \times S L(2, \mathbb{C})^{k}$ acting on $\left(\mathbb{C}^{2}\right)^{\otimes k}$. We can compute the dimension of the $\mathfrak{S}_{k}$-invariants as follows:

Proposition 5.2. The dimension of the space of $\mathfrak{S}_{k} \ltimes G$-invariants in $\mathcal{R}_{2, k, 4}$ is $M_{k}=$ $\left[\frac{k}{6}\right]+r_{k}$ where $r_{k}=0$ if $k \equiv 1 \bmod 6$, or $r_{k}=1$ otherwise. Furthermore the algebra $\oplus_{k} \mathcal{R}_{2, k, 4}^{\mathfrak{S}_{k} \times G}$ is the polynomial algebra $\mathbb{C}\left[P_{2,2}^{2}, H_{2,3}\right]$.

Proof. We have isomorphisms $\mathcal{R}_{2, k, 4}^{\mathfrak{S}_{k} \times G} \simeq\left(E^{\otimes k}\right)^{\mathfrak{S}_{k} \times \mathfrak{S}_{3}} \simeq S^{k}(E)^{\mathfrak{S}_{3}}$ since the representation of $\mathfrak{S}_{4}$ on $E$ factors through $\mathfrak{S}_{3}$. Thus the algebra $\oplus_{k} \mathcal{R}_{2, k, 4}^{\mathfrak{S}_{k} \times G}$ identifies with $S(E)^{\mathfrak{S}_{3}}$. Now $S(E)^{\mathfrak{S}_{3}}$ is the algebra of $\mathfrak{S}_{3}$-invariant polynomial functions on traceless $3 \times 3$ diagonal matrices, and so is a polynomial algebra on the functions $A \mapsto \operatorname{Tr}\left(A^{2}\right)$ and $A \mapsto \operatorname{Tr}\left(A^{3}\right)$. These invariants correspond (up to scaling) to $P_{2,2}^{2}$ and $H_{2,3}$. The formula for the dimension follows easily.

For instance, we have: $M_{1}=0, M_{k}=1$ for $2 \leq k \leq 5$, and $M_{6}=2$. We remark that by replacing $S(E)^{\mathfrak{S}_{3}}$ by $\wedge(E)^{\mathfrak{S}_{3}}$, it is easy to prove that the sign representation of $\mathfrak{S}_{k}$ does not occur in $\left(E^{\otimes k}\right)^{\mathfrak{S}_{4}}$ for any $k \geq 2$.

We can determine the $\mathfrak{S}_{k}$-representation on $\mathcal{R}_{2, k, 4}^{G}$ for small $k$ by explicit trace computations. For $k=2$ and $k=3$ we have the trivial 1-dimensional representation. For $k=4$, we find $\mathcal{R}_{2,4,4}^{G}$ is the direct sum $E_{[4]} \oplus E_{[2,2]}$. The trivial representation $E_{[4]}$ of $\mathfrak{S}_{4}$ is spanned by $P_{2,4}^{2}$, while the 2-dimensional representation $E=E_{[2,2]}$ is spanned by the determinants $\Delta(i j k l)$ introduced in $[B]$. Here $(i j k l)$ is a permutation of (1234). Given a tensor state $u \in\left(\mathbb{C}^{2}\right)^{\otimes 4}$, we can view it as an element $v$ of $\mathbb{C}^{4} \otimes \mathbb{C}^{4}$, where the first (resp. second) $\mathbb{C}^{4}$ is the tensor product of the $i$-th and $j$-th copies of $\mathbb{C}^{2}$ (resp. of the $k$-th and $l$-th copies). Then $\Delta(i j k l)(u)$ is the determinant of $v$. As shown in [B], the $\Delta(i j k l)$ span the representation $E$ of $\mathfrak{S}_{4}$. The significance of the $\Delta(i j k l)$ is that their vanishing describes the closure of the set of tensor states in $\left(\mathbb{C}^{2}\right)^{\otimes 4}$ of rank $\leq 3$. For $k=5$ the representation $\mathcal{R}_{2,5,4}^{G}$ of $\mathfrak{S}_{5}$ is $E_{[5]} \oplus E_{[2,1,1,1]}$.

\section{References}

[B] J-L. Brylinski, Algebraic measures of entanglement, quant-ph 0008031

[C-K-W] V. Coffman, J. Kundu and W. K. Wootters, Distributed Entanglement, preprint quant-ph/9907047 
[G-K-Z] I.M. Gelfand, M. Kapranov and A. Zelevinsky, Discriminants, Resultants and Multidimensional Determinants, Birkhäuser (1991)

[G-R-B1] M. Grassl, M. Rötteler and T. Beth, Computing Local Invariants of QuantumBit Systems, Phys. Review A 58 no. 3 (1998), 1833-1839; also on the Arxiv as quant-ph/9712040

[G-R-B2] M. Grassl, M. Rötteler and T. Beth, Description of Multi-Particle Entanglement through Polynomial Invariants, Talk of M Grassl at the Isaac Newton Institute for Mathematical Sciences in July 1999, available on the web as http://iakswww.ira.uka.de/home/grassl/publications.htm]

[L-P] N. Linden and S. Popescu, On Multi-Particle Entanglement, Forts. der Physik 46 (1998), no. 4-5, 567-578, also on the Arxiv as quant-ph/9711016

[R] E. Rains, Polynomial Invariants of Quantum Codes, EEE Trans. on Information Th. 46 no. 1 (2000), 54-59 